\title{
Fast robber in planar graphs ${ }^{\star}$
}

\author{
Nicolas Nisse ${ }^{1}$ and Karol Suchan ${ }^{12}$ \\ nnisse@dim.uchile.cl, karol@suchan.info \\ 1 Departamento de Ingeniería Matemática, Universidad de Chile, Santiago, Chile \\ ${ }^{2}$ Faculty of Applied Mathematics, AGH - University of Science and Technology, Cracow, Poland
}

\begin{abstract}
In the cops and robber game, two players play alternately by moving their tokens along the edges of a graph. The first one plays with the cops and the second one with one robber. The cops aim at capturing the robber, while the robber tries to infinitely evade the cops. The main problem consists in minimizing the number of cops used to capture the robber in a graph. This minimum number is called the cop-number of the graph. If the cops and the robber have the same velocity, $3+\frac{3}{2} g$ cops are sufficient to capture one robber in any graph with genus $g$ (Schröder, 2001). In the particular case of a grid, 2 cops are sufficient.

We investigate the game in which the robber is slightly faster than the cops. In this setting, we prove that the cop-number of planar graphs becomes unbounded. More precisely, we prove that $\Omega(\sqrt{\log n})$ cops are necessary to capture a fast robber in the $n \times n$ square-grid. This proof consists in designing an elegant evasion-strategy for the robber. Then, it is interesting to ask whether a high value of the cop-number of a planar graph $H$ is related to a large grid $G$ somehow contained in $H$. We prove that it is not the case when the notion of containment is related to the classical transformations of edge removal, vertex removal, and edge contraction. For instance, we prove that there are graphs with cop-number at most 2 and that are subdivisions of arbitrary large grid. On the positive side, we prove that, if $H$ planar contains a large grid as an induced subgraph, then $H$ has large cop-number. Note that, generally, the cop-number of a graph $H$ is not closed by taking induced subgraphs $G$, even if $H$ is planar and $G$ is an distance-hereditary induced-subgraph.

Keywords: Cops and robber, planar graph, minor, subdivision, grid.
\end{abstract}

\section{Introduction}

Introduced by Nowakowsky and Winkler [NW83], and by Quilliot [Qui83], cops and robber game is a two player game in a graph $G$ (see [Als04] for a survey). The first player, $\mathcal{C}$, plays with the cops that are aiming at capturing the robber, played by the second player $\mathcal{R}$. First, $\mathcal{C}$ chooses a subset of vertices of $G$ and places his cops on these vertices. Then, $\mathcal{R}$ places his robber on some vertex of $G$. Then, $\mathcal{C}$ and $\mathcal{R}$ play alternately. At each step, $\mathcal{C}$ chooses a subset of his cops and move each of them along some path of length at most $v_{c o p} \geq 1$ edges. Then, $\mathcal{R}$ moves his robber along some path of length at most $v_{\text {robber }} \geq 1$ edges. Note that both players have perfect knowledge of the position(s) of their adversary. The robber is caught as soon as it occupies the same vertex as a cop. The purpose of $\mathcal{C}$ is to capture the robber, while $\mathcal{R}$ tries to infinitely avoid being caught. In the following, we refer to $v_{c o p}$ and $v_{\text {robber }}$ as the cops' speed and the robber's speed, respectively. For any graph $G$, the $(p, q)$-cop-number, denoted $c_{p, q}(G)$, is the smallest number of cops with speed $p$ sufficient to capture any robber with speed $q$ in $G$.

The case $p=q=1$ has received particular attention in the literature, and $c_{1,1}(G)$ is generally called the cop-number of the graph $G$. The main result in [NW83,Qui83] is a characterization of the graphs with cop-number one, called cop-win graphs. In particular, trees, chordal graphs and, more generally, graphs with chordality at most 4 are cop-win [Far87,AF88,Che97]. This characterization also allowed Hahn and MacGillivray [HM06] to design an algorithm deciding in time $O\left(n^{k}\right)$ if the cop-number of a $n$-node graph is at most $k \geq 1$ (see also [BI93]). Goldstein and Reingold [GR95] prove that the problem of computing the cop-number of a directed graph (in this setting, the cops and the robber are constrained to follow the orientation of the arcs)

\footnotetext{
* Authors acknowledge the support of CONICYT via Anillo en Redes ACT08
} 
is EXPTIME-complete. This problem is EXPTIME-complete as well in undirected graph when cops and robber are given their initial positions [GR95].

¿From a combinatorial point of view, the cop-number of bounded genus graphs has been widely studied. In [AF84], Aigner and Fromme proved that the cop-number of any planar graph is at most three. In particular, the cop-number of any grid is two. The result of Aigner and Fromme is based on the simple following Proposition 1.

Proposition 1. [AF84] In any graph $G$ and for any shortest path $P$ of $G$, after a finite number of steps, a single cop can prevent the robber with speed one from entering $P$.

Then, Aigner and Fromme [AF84] prove that it is possible to recursively divide any planar graph using three shortest paths chosen in such a way that the area accessible to the robber only decreases. Using the same kind of techniques, Quilliot [Qui85] proves that the cop-number of any graph with genus $g \geq 0$ is at most $3+2 g$. Schröder [Sch01] improves this bound to $3+\frac{3}{2} g$. Proposition 1 is also essential in the proof of the fact the cop-number of any $H$-minor-free graph is upper bounded by the number of edges of $H$ [And86].

It is noticeable that very few lower bounds of the cop-number of graphs have been proved. Aigner and Fromme [AF84] prove that the cop-number of any graph with girth at least 5 is lower bounded by its minimum degree. Frankl [Fra87] improves this bound to $d^{t}$ for any graph with girth at least $8 t-3$ and minimum degree $d+1$. Note also that, for any $k \geq 3$ and $n \geq 1$, it exists a $k$-regular graph with cop-number at least $n$ [And84].

We investigate the cops and robber game in planar graphs when the robber is slightly faster than the cops, i.e., $v_{\text {robber }}>v_{\text {cop }}$. It is easy to be convinced that Proposition 1 becomes false as soon as the robber is faster than the cops. In particular, we prove that allowing the robber to be faster than the cops may drastically increase the number of cops necessary to capture it in a square-grid. We then generalize this result to a larger class of planar graphs. More precisely, we propose a certificate that is sufficient to decide that "many" cops are necessary to capture a fast robber. We leave as an open question, if our certificate is also a necessary condition.

For ease of description, in this paper, we consider that the robber's speed is two while the cops' speed is one, and we refer to $c_{1,2}(G)$ as the cop-number of the graph $G$. However our results can easily be generalized for any $v_{\text {robber }} / v_{\text {cop }}>1$.

\subsection{Our results}

Our main result consists in proving that the cop-number of square-grids is not bounded. We prove that the cop-number of a $n \times n$ square-grid is at least $\Omega(\sqrt{\log n})$. The proof is constructive since we give a simple and explicit evasion-strategy for the robber. More precisely, we prove that, for any $k \geq 1$, there are two constants $a>0$ and $b>2$, such that, one robber with speed 2 can infinitely evade $k$ cops with speed one in any $n \times n$ square-grid with $n \geq 4 a^{k} b^{k(k+1) / 2}=f(k)$.

A natural question is then to ask whether this lower bound still holds for planar graphs somehow containing a large grid. In other words, is a high value of the cop-number of a planar graph $H$ related to a large grid $G$ somehow contained in $H$ ? On the negative side, the classical transformations of edge removal, vertex removal, and edge contraction do not preserve "small" cop-number. For instance, for any $k \geq 1$, we design a subdivision $H$ of a $n \times n$ square-grid with $n \geq f(k)$, such that the cop-number of $H$ is at most 2 . The converse also holds: we prove that the cop-number of a planar graph may drastically decrease by contracting edges incident to degree-2 vertices. This confirms the intuition according to which the cop-number of a graph is more related to the distances rather than to the connectivity of the graph.

On the other hand, we prove that if $H$ planar contains a large grid as an induced subgraph, then $H$ has large cop-number. More precisely, any planar graph $H$ that contains a $n \times n$ squaregrid $G$ with $n \geq 2 f(k)$ as an induced subgraph has cop-number at least $k$. Note that this 
latter result is not trivial because the cop-number of a graph is generally not closed by taking induced subgraphs, even if $H$ is planar and $G$ is a distance-hereditary induced-subgraph. Indeed, consider the cycle $C_{4}$, and let $H$ be the graph obtained from $C_{4}$ by adding a universal vertex. The cop-number of $H$ equals one, whereas it equals two in $C_{4}$.

\section{Fast Robber in large grid}

This section is devoted to prove the following theorem.

Theorem 1. For any grid $G$ of size $n, c_{1,2}(G)=\Omega(\sqrt{\log (n)})$.

To prove Theorem 1, we propose an evasion strategy for the robber. This strategy is formally described in section 2.2. The proof of its correctness mainly follows Lemma 3. Lemmata 1 and 2 are technical results that allow to prove Lemma 3.

\subsection{Definitions}

We consider a robber that is slightly faster than the cops running in a square grid. A square grid $G$ on $n \times n$ vertices is the graph where the vertices can be naturally assigned to the points of positive integer coordinates in the square $n \times n$ of the plane, with edges joining each vertex to its closest neighbors (with respect to the Euclidean metric). We say that the size of $G$ is $n$. In order to prove that the number of cops needed to capture the robber is unbounded, for each number $k$ of cops, we will construct a grid $G(k)$ of size $f(k)$, and a corresponding strategy, by which the robber can infinitely evade the cops.

Given the number of cops $k$ and the corresponding grid $G=G(k)$, a key to our analysis lies in fixing a recursive partition of the grid into gradually smaller subgrids of levels $k$ down to 0 . Each level $i$ corresponds to the game played on a subgrid of size size $e_{i}$, with only $i$ cops taken into consideration. At each step $s$, the subgrid of level $i$, or $i$-subgrid, currently occupied by the robber is denoted by $R_{s}^{i}$. Let us fix an ordering of the cops: $\operatorname{cop}_{1}, \ldots, \operatorname{cop}_{k}$. The sizes of subgrids are chosen such that there is a strategy allowing the robber to successfully evade $i$ cops in the $i$-subgrid $R^{i}$ around him, and to move to neighboring $i$-subgrids fast enough not to let other cops enter into $R^{i}$. And that is for each $i$ between 0 and $k$. Let us introduce some notation that we use in order to describe the above mentioned strategy on the graph $G=G(k)$.

$z o o m=\left(z o o m_{1}, \cdots, z_{o o m}\right)$ is a sequence of scaling factors, that is, an $i$-subgrid contains $z o o m_{i} \times z_{o o m}$ vertex disjoint $(i-1)$-subgrids. This means that size $e_{i}$ is equal to $z o o m_{i} \times s_{i z} e_{i-1}$, where we fix $s i z e_{0}=2$ as a starting point. We say that an $i$-subgrid $H^{i}$ is adjacent to a $j$-subgrid $F^{j}$ if there is an edge in $G$ incident to a vertex in each of them. When $i$ is clear from the context, an $(i-1)$-subgrid relative to an $i$-subgrid is called a square on a board. A path of squares is a sequence of squares such that any square is adjacent to its predecessor, and its length is simply the number of squares. In this way, we can notice a fractal-like structure of $G$, with the grid topology of squares on boards of corresponding levels. Let us introduce a coordinate system for subgrids at each level. The coordinates of an $i$-subgrid $H$ are $\left(a b s_{H}, o r d_{H}\right)$, which correspond to the row (bottom-up) and column (left-right) occupied by $H$ in the partition of $G$ into subgrids of size $e_{i}$. In other words, a vertex $v$ is in $H$ iff the abscissa of $v$ is between $\left(a b s_{H}-1\right) * s i z e_{i}+1$ and $a b s_{H} * s i z e_{i}$, and the ordinate of $v$ is between $\left(\operatorname{ord}_{H}-1\right) * \operatorname{size}_{i}+1$ and $\operatorname{ord}_{H} * \operatorname{size}_{i}$.

margin $=\left(\operatorname{margin}_{1}, \cdots, \operatorname{margin}_{k}\right)$ is a sequence of safety distances. Given an $i$-subgrid $H^{i}$, we note by around $\left(H^{i}\right)$ the subgrid induced by the $i$-subgrids that are near $H^{i}$. More formally, $\operatorname{around}\left(H^{i}\right)$ is the subgrid induced by the $i$-subgrids $H$, such that $\left|\operatorname{ord}_{H^{i}}-\operatorname{ord}_{H}\right| \leq 1$ and $\left|a b s_{H^{i}}-a b s_{H}\right| \leq 1$. Similarly, we define the margin of $H^{i}$, denoted by $\operatorname{margin}\left(H^{i}\right)$, as the subgrid induced by the $i$-subgrids $H$, such that $\left|\operatorname{ord}_{H^{i}}-\operatorname{ord}_{H}\right| \leq m_{i}$ and $\left|a b s_{H^{i}}-a b s_{H}\right| \leq m_{i}$. 
For any $i$, a configuration in which $\operatorname{cop}_{i}$ is outside the subgrid $\operatorname{around}\left(R^{i-1}\right)$ (i.e., cop $i$ does not occupy any vertex of it), where $R^{i-1}$ is the square occupied by the robber, is a valid position at level $i$. If moreover, the cop $\operatorname{cop}_{i}$ is also outside $\operatorname{margin}\left(R^{i-1}\right)$ and $\operatorname{margin}\left(R^{i-1}\right)$ is a subgraph of $R^{i}$, the position of the robber is called a nice position at level $i$.

Definition 1. The robber occupies an i-nice position if it occupies a nice position at level $j$, for all $1 \leq j \leq i$.

Suppose the robber is in a nice position at level $i$. If $\operatorname{cop}_{i}$ occupies a square adjacent to the margin, we say that the cop is blocking a side. If $\operatorname{cop}_{i}$ occupies a square in a corner (adjacent to two other squares blocking different sides), we say that the cop is blocking a corner.

detour $=\left(\right.$ detour $_{1}, \cdots$, detour $\left._{k}\right)$ is a sequence of extra distances. At level $i$, detour $r_{i}$ is an upper bound on the additional distance that the robber needs to travel in order to evade $\operatorname{cop}_{i}$. More precisely, starting from a nice position at level $i$, the length of the path of squares that the robber will follow to go into a nice position in a neighboring board is upper bounded by $z_{o o m}+$ detour $_{i}$. Notice that $z o o m_{i}$ is the minimum number of squares that the robber needs to cross in order to get from the left extreme (resp., up extreme) of a board $H^{i}$ to the board $F^{i}$ next to the right (resp., down) of $H^{i}$.

time $=\left(\right.$ time $_{0}, \cdots$, time $\left._{k}\right)$ is a sequence of numbers of rounds. At level $i, t_{i m e}$ is an upper bound on the time needed by the robber in order to get from a nice position on a board $H^{i}$ to a nice position on a neighboring board $F^{i}$. Moreover, we set the sequence velocity $=$ $\left(\right.$ velocity $_{0}, \cdots$, velocity $y_{k}$, with velocity $y_{i}=$ size $_{i} /$ time $_{i}$, as the "relative" speed of the robber at level $i$. Since the robber has speed velocity $y_{0}=2$, which is its "absolute" speed, and size $_{0}=2$, we get time $_{0}=1$.

\subsection{Informal description of the robber's strategy}

In this section we give an intuitive description of the robber's strategy in order to explain the relations between the sequences defined in the previous section.

Let $1 \leq i \leq k$. Recall that a square denotes an $(i-1)$-subgrid and a board denotes an $i$-subgrid.

Let us first describe our induction hypothesis at level $i-1$. Let $R^{i-1}$ be the square occupied by the robber. We assume that if all of the cops $\operatorname{cop}_{j}, i-1<j$, remain outside $\operatorname{around}\left(R^{i-1}\right)$, and if the initial position of the robber is $(i-1)$-nice, then the robber can reach an $(i-1)$-nice position in any square adjacent to $R^{i-1}$, in at most time $_{i-1}<$ size $_{i-1}$ rounds. Let us describe the robber's strategy that ensures that the induction hypothesis remains valid at the level $i$.

Let $R^{i}$ be the board that is occupied by the robber. We assume that all of the cops $\operatorname{cop}_{j}$, $j>i$, remain outside around $\left(R^{i}\right)$ during the whole game that we will describe. Moreover, let us assume that, for any $1 \leq j \leq i$, the initial position of the robber is nice at level $j$. In other words, the robber occupies an $i$-nice position. In particular, it means that $\operatorname{cop}_{i}$ is outside the subgrid $\operatorname{margin}\left(R^{i-1}\right)$ and margin $\left(R^{i-1}\right)$ is a subgraph of $R^{i}$, where $R^{i-1}$ denotes the $(i-1)$-subgrid initially occupied by the robber. Let $D^{i}$ be a board that is a neighbor of $R^{i}$. We describe a strategy for the robber that ensures that (1) the robber reaches an $i$-nice position in $D^{i}$ in at ${\text { most } \text { time }_{i}<\text { size }_{i} \text { steps, and (2) cop }}_{i}$ remains outside around $\left(R^{i-1}\right)$ during the whole game.

For ease of description, we assume that $D^{i}$ is below $R^{i}$ (i.e., $D^{i}$ has smaller ordinate than $\left.R^{i}\right)$. This strategy is depicted in Figure 1. In Figures 1(a), 1(b), and 1(c), the hatched zone corresponds to the path of squares covered by the robber during the game.

At each step $s \geq 0$ of the game, let $R_{s}^{i-1}$ and $C_{s}^{i-1}$ be the squares occupied by the robber and $c^{c o p} p_{i}$ respectively. Roughly speaking, the strategy consists in the following. While $\operatorname{cop}_{i}$ is outside $\operatorname{margin}\left(R_{s}^{i-1}\right)$ and does not block neither a side nor a corner of $\operatorname{margin}\left(R_{s}^{i-1}\right)$, the robber goes 


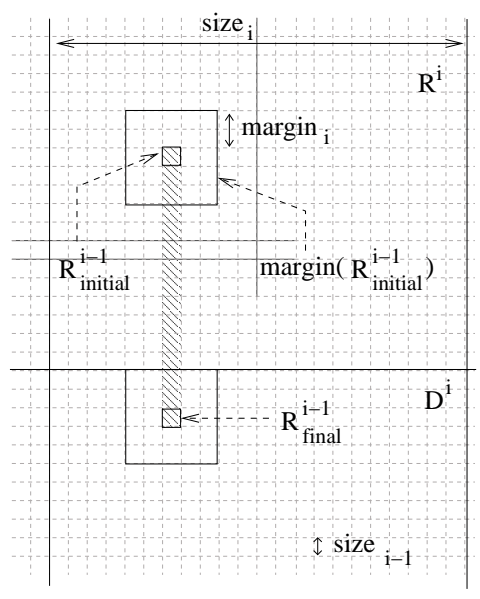

(a) Illustration of Strategy 1

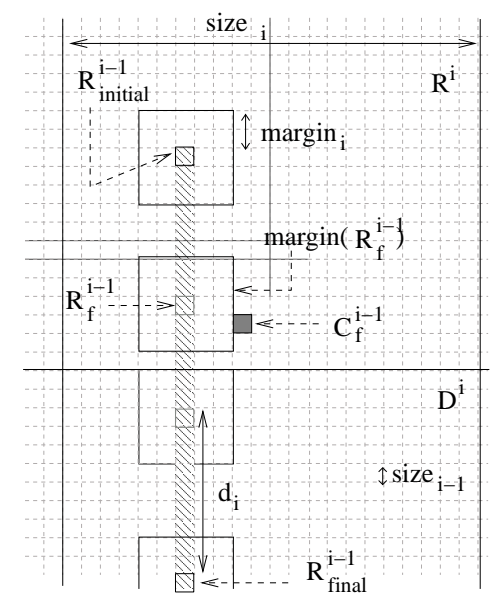

(b) Illustration of Strategy 2

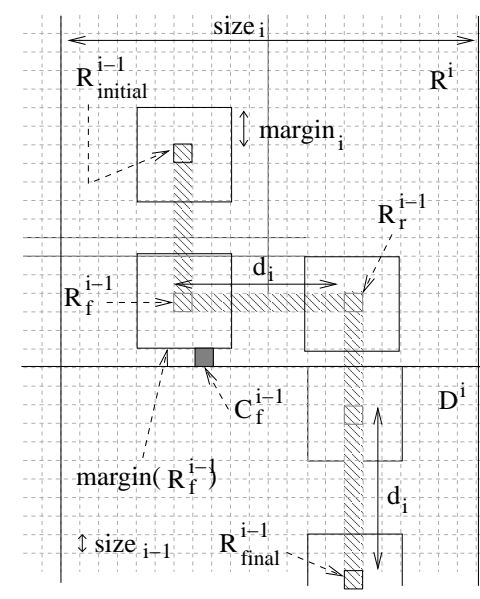

(c) Illustration of Strategy 3

Fig. 1. Strategy performed by the robber if (a) $\operatorname{cop}_{i}$ never blocks a side of $\operatorname{margin}\left(R_{s}^{i}\right),(\mathrm{b}) \operatorname{cop}_{i}$ blocks any but the bottom side of $\operatorname{margin}\left(R_{s}^{i}\right)$, and (c) $\operatorname{cop}_{i}$ blocks the bottom side of $\operatorname{margin}\left(R_{s}^{i}\right)$.

down, that is, it goes to the square $H^{i-1}$ that is the below-neighbor of $R_{s}^{i-1}$. By applying the induction hypothesis, the robber reaches an $(i-1)$-nice position in $H^{i-1}$ in at most time $e_{i-1}$ steps. If, performing that way, the robber reaches a square $H^{i-1}$ such that $\operatorname{margin}\left(H^{i-1}\right)$ is a subgraph of $D^{i}$, we are done. Moreover, it has taken at most $z o o m_{i} * t i m e_{i-1}$ steps. This strategy is illustrated in Figure 1(a). If at some step $f$ of the game, $c_{i}$ is blocking a side or a corner of $\operatorname{margin}\left(R_{f}^{i-1}\right)$, we consider different cases according to which side or corner of $\operatorname{margin}\left(R_{f}^{i-1}\right)$ is blocked.

- Let us first assume that $\operatorname{cop}_{i}$ blocks a side or a corner above $\operatorname{margin}\left(R_{f}^{i-1}\right)$. That is, $C_{f}^{i-1}$ has greater ordinate than any square in margin $\left(R_{f}^{i-1}\right)$. Then, the strategy remains the same: the robber goes down (cf. Figure 1(a)). The robber traverses a square in at most time $_{i-1}<$ size $_{i-1}$ steps, whereas $\operatorname{cop}_{i}$ needs size $e_{i-1}$ steps. By Lemma 1, size $e_{i-1}>t_{i-1}$. Therefore, each time the robber moves to a new square $R_{s}^{i-1}, \operatorname{cop}_{i}$ is outside $\operatorname{margin}\left(R_{s}^{i-1}\right)$. In particular, this is the case at the step when $\operatorname{margin}\left(R_{s}^{i-1}\right)$ is contained in $D^{i}$ for the first time. Then the strategy achieves.

- Let us now assume that $\operatorname{cop}_{i}$ blocks a side or a corner at the left (resp. at the right) of $\operatorname{margin}\left(R_{f}^{i-1}\right)$. That is, $C_{f}^{i-1}$ has smaller (resp., greater) abscissa than any square in $\operatorname{margin}\left(R_{f}^{i-1}\right)$. Again, the strategy consists in going down. However, this time, after the first step when margin $\left(R_{s}^{i-1}\right)$ is a subgraph of $D^{i}$, the robber continues going down along a path of $d_{i}$ extra squares in $D^{i}$. This is because the cop may enter in $\operatorname{margin}\left(R_{s}^{i-1}\right)$ during this passage, and we want to have it outside the margin in the end. This strategy is illustrated in Figure 1(b). $d_{i}$ corresponds to an extra distance that the robber must cover in order to avoid $\operatorname{cop}_{i}$. detour $i$ will be taken equal to an upper bound of this extra distance in any of the strategies described below. For our strategy to be valid, we must ensure two properties. First, in order to apply the induction hypothesis, $с_{0} p_{i}$ must permanently remain outside $\operatorname{around}\left(R_{s}^{i-1}\right)$. Second, at some step $s \leq$ time $_{i}$, the robber must reach an $i$-nice position in $D^{i}$, that is, $\operatorname{cop}_{i}$ must be outside $\operatorname{margin}\left(R_{s}^{i-1}\right)$ while $\operatorname{margin}\left(R_{s}^{i-1}\right)$ is a subgraph of $D^{i}$. In order to ensure the above two properties, we set several inequalities between size $_{i-1}$, zoom $_{i}$, margin $_{i}$, detour $_{i}$, time $_{i-1}$, velocity $_{i-1}$ and time $_{i}$.

- For the first property to be satisfied, it is sufficient to ensure that, if $C_{f}^{i-1}$ is blocking the left-bottom corner of $\operatorname{margin}\left(R_{f}^{i-1}\right)$ and $\operatorname{cop}_{i}$ goes to the right while the robber is 
going down, then $\operatorname{cop}_{i}$ cannot enter around $\left(R_{s}^{i-1}\right)$. Indeed, one can observe that the cop occupying this position yields the worst possible case of blocking a side or a corner. Let $N$ be the minimum number of steps that are necessary for the cop to intercept the robber, and let $M$ be the maximum number of steps that are necessary for the robber to cross the place of this hypothetical interception. In other words, we want that $M<N$. Recall that $s_{i z e_{i-1}}$ is the minimum number of steps for a cop to traverse a square (from one of its sides to cross the opposite one), whereas time ti-1 $_{1}$ is the maximum number of steps for the robber to cover the same distance. By looking at Figure 2(a), it is easy to be convinced that $N>\left(\operatorname{margin}_{i}-1\right)$ size $_{i-1}$, and $M<\left(4+\operatorname{margin}_{i}\right)$ time $_{i-1}$. In Figure 2(a), $M_{1}=4+\operatorname{margin}_{i}$ and $N_{1}=\operatorname{margin}_{i}-1$. Hence, we get our first inequality. For any $i, 1 \leq i \leq k$ :

$$
\operatorname{margin}_{i} \geq\left\lceil\frac{4+\text { velocity }_{i-1}}{\text { velocity }_{i-1}-1}\right\rceil
$$

- For the second property to be satisfied, it is sufficient to ensure that, if the step $f$ is such that the squares of $\operatorname{margin}\left(R_{f}^{i-1}\right)$ with the greatest ordinate are still in $R^{i}$ and all the other squares of it are in $D^{i}$, and $C_{f}^{i-1}$ is the left-bottom corner of $\operatorname{margin}\left(R_{f}^{i-1}\right)$, then $\operatorname{cop}_{i}$ is above $\operatorname{margin}\left(R_{h}^{i-1}\right)$ at the last step $h$ of the game, and $\operatorname{margin}\left(R_{h}^{i-1}\right)$ is a subgraph of $D^{i}$. Again, this position of $R_{f}^{i-1}$ leads to the worse possible configuration of this case. Let $N$ be the minimum number of steps that are necessary for the cop to reach $\operatorname{margin}\left(R_{h}^{i-1}\right)$, and let $M$ be the maximum number of steps that are necessary for the robber to reach $R_{h}^{i-1}$. Again, we want that $M<N$. Moreover, $h \leq t_{i}$. Looking at Figure $2(\mathrm{~b})$, it is easy to be convinced that $N>\left(d_{i}-2 * \operatorname{margin}_{i}-2\right) \operatorname{size}_{i-1}$, and $M<d_{i} * t_{i m e} e_{i-1}$. Hence, we get our second equation. For any $i, 1 \leq i \leq k$ :

$$
d_{i} \geq\left\lceil\frac{\left(2 * \text { margin }_{i}+2\right) \text { velocity }_{i-1}}{\text { velocity }_{i-1}-1}\right\rceil
$$

For the final position of the robber to be nice, we also need $\operatorname{margin}\left(R_{h}^{i-1}\right)$ to be a subgraph of $D^{i}$, that is:

$$
d_{i}+2 \operatorname{margin}_{i}+1<z_{\text {oom }_{i}}
$$

Finally, the whole game must take at most time $_{i}<$ size $_{i}$ steps, therefore:

$$
\left(\text { zoom }_{i}+d_{i}\right) \text { time }_{i-1}<\left(\text { zoom }_{i}+\text { detour }_{i}\right) \text { time }_{i-1} \leq \text { time }_{i}<\text { size }_{i}
$$

- It remains the case when $\operatorname{cop}_{i}$ blocks a side below $\operatorname{margin}\left(R_{f}^{i-1}\right)$. That is, $C_{f}^{i-1}$ has smaller ordinate than any square in margin $\left(R_{f}^{i-1}\right)$. In this case, the robber chooses the right side, if $R_{f}^{i-1}$ is closest to this side of $R^{i}$, and the left side otherwise. W.l.o.g., let the robber choose the right side. Then, the robber first goes to the right, along a path of $d_{i}$ squares. Let $R_{r}^{i-1}$ be the last of these squares, at which the robber arrives at step $r$. Note that, by Inequality $1, \operatorname{cop}_{i}$ never enters around $\left(R_{s}^{i-1}\right)$ during this phase. Moreover, by Inequality 2 , at step $r, \operatorname{cop}_{i}$ is to the left of margin $\left(R_{r}^{i-1}\right)$. Starting from step $r$, the strategy is the same as in the previous case: the robber goes down, and after the first step when $\operatorname{margin}\left(R_{s}^{i-1}\right)$ is a subgraph of $D^{i}$, the robber continues going down along a path of $d_{i}$ extra squares in $D^{i}$. This strategy is illustrated in Figure 1(c). Again, by applying Inequalities 1 and 2, we get that $\operatorname{cop}_{i}$ never enters around $\left(R_{s}^{i-1}\right)$ during the whole game, and, at the last step $h, \operatorname{cop}_{i}$ is outside $\operatorname{margin}\left(R_{h}^{i-1}\right)$. In order to ensure $\operatorname{margin}\left(R_{h}^{i-1}\right)$ to be a subgraph of $D^{i}$, we need the following inequality:

$$
d_{i}+2 * \operatorname{margin}_{i}+1<\text { zoom }_{i} / 2
$$




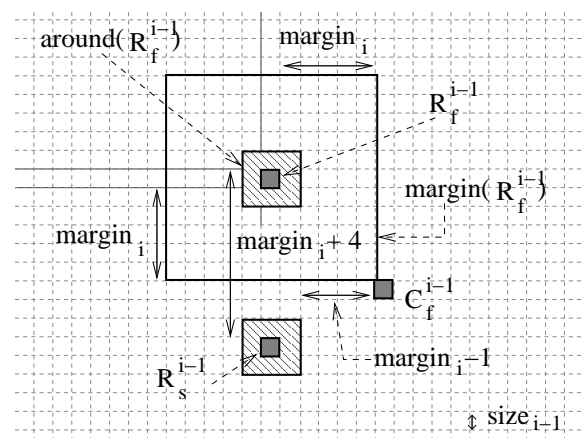

(a) margin $\left._{i}-1\right)$ size $_{i-1} \geq\left(4+\right.$ margin $\left._{i}\right)$ time $_{i-1}$

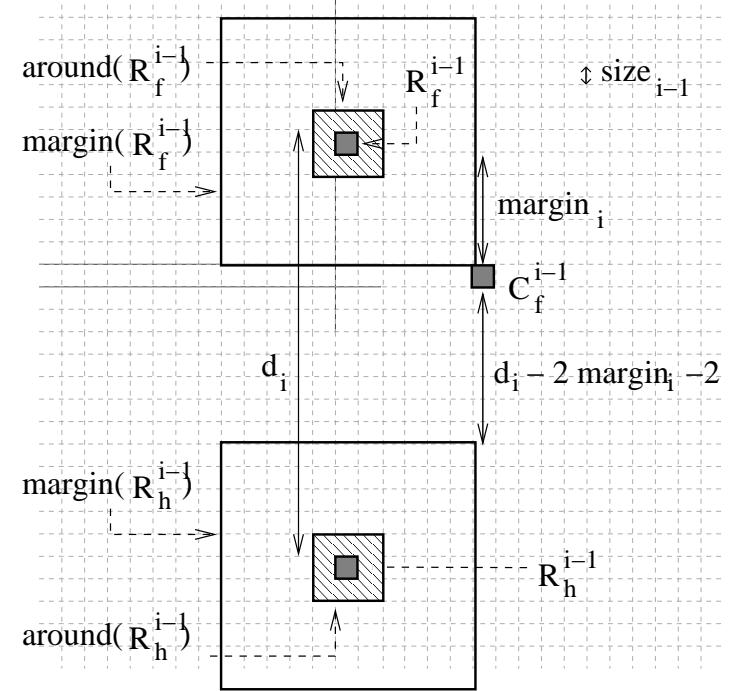

(b) $\left(d_{i}-2 * \operatorname{margin}_{i}-2\right)$ size $_{i-1} \geq d_{i} *$ time $_{i-1}$

Fig. 2. Illustration of Inequality 1 and 2: (a) $\operatorname{cop}_{i}$ must never enter in $\operatorname{around}\left(R_{s}^{i}\right)$, and (b) the robber must reach a nice position, i.e., $\operatorname{cop}_{i}$ must not enter in $\operatorname{margin}\left(R_{h}^{i}\right)$.

Finally, the whole game must take at most time $_{i}<$ size $_{i}$ steps, therefore:

$$
\left(\text { zoom }_{i}+2 d_{i}\right) \text { time }_{i-1} \leq\left(\text { zoom }_{i}+\text { detour }_{i}\right) \text { time }_{i-1} \leq \text { time }_{i}<\text { size }_{i}
$$

In the following, we turn Inequalities 1 and 2 into equalities, we set $\operatorname{detour}_{i}=2 d_{i}$, and we prove that, for a sequence zoom well chosen, Inequalities 3 and 4 are satisfied.

\subsection{Proof of Theorem 1}

We first prove that there are two constants $a>0$ and $b>2$, such that to set $z o o m_{i}=a b^{i}$ (for all $i \leq k$ ) ensures that Inequalities 3 and 4 are valid.

Let $k \geq 1$ and velocity $y_{0}=2$. We will now precisely define the sequence $z o o m$, and define the relations between the sequences zoom, margin, detour, time, size and velocity. For any $1 \leq i \leq k$, let us turn Inequalities 1 and 2 into equalities:

$$
\text { margin }_{i}=\left\lceil\frac{4+\text { velocity }_{i-1}}{\text { velocity }_{i-1}-1}\right\rceil, \text { and }, \text { detour }_{i}=2 d_{i}=2 *\left\lceil\frac{\left(2 * \text { margin }_{i}+2\right) \text { velocity }_{i-1}}{\text { velocity }_{i-1}-1}\right\rceil .
$$

We also set:

$$
\text { time }_{i}=\left(\text { zoom }_{i}+\text { detour }_{i}\right) \text { time }_{i-1}
$$

¿From Equations 5, 6 and the fact that velocity $y_{i}=$ size $_{i} /$ time $_{i}$, we get that velocity $i=$ $\frac{\text { zoom }_{i}}{\text { zoom }_{i}+\text { detour }_{i}} *$ velocity $_{i-1} \geq \beta_{i} *$ velocity $_{i-1}$, where $\beta_{i}$ is defined by:

$$
\beta_{i}=\frac{z_{\text {oom }_{i}}}{z \text { oom }_{i}+2 *\left(\frac{\left(2 *\left(\frac{4+\text { velocit }_{i-1}}{\text { velocit }_{i-1}-1}+1\right)+2\right) \text { velocity }_{i-1}}{\text { velocit }_{i-1}-1}+1\right)}=\frac{\text { ooom }_{i}}{z \text { oom }_{i}+\frac{2+4 * \text { velocit }_{i-1}+14 * \text { velocity }_{i-1}^{2}}{\left(\text { velocity }_{i-1}-1\right)^{2}}}
$$

Finally, let us assign some values to the sequence zoom, in order to satisfy Inequalities 3 and 4 . For this purpose, let us set $2>\alpha>1$, and let $a=\left\lceil\frac{20}{(\alpha-1)^{2}}\right\rceil *\left\lceil\frac{2}{\ln (\text { velocityo } / \alpha)}\right\rceil$ and let $b$ be an integer such that $b>\max \left\{2, \frac{\ln (\text { velocity } / \alpha)}{2}\right\}$. For any $1 \leq i \leq k$, we set

$$
z o o m_{i}=a b^{i} \text {. }
$$


Lemma 1. Inequality 4 is satisfied. That is, for any $0 \leq i \leq k$, velocity $_{i}=$ size $_{i} /$ time $_{i}>1$.

Proof. We prove by induction on $i, 0 \leq i \leq k$, that $2 \geq$ velocity $_{i}>\alpha$. It is straightforward for $i=0$. Let $i>0$, and let us assume that the result is valid for any $j<i$. velocity $i=$ velocity $y_{0} * \prod_{j=1}^{i} \beta_{j}$, thus we need to prove that $\prod_{j=1}^{i} \beta_{j}>\alpha /$ velocity $y_{0}$. Actually, we prove that $1 / \prod_{j=1}^{i} \beta_{j}<$ velocity $_{0} / \alpha$. By the induction hypothesis, we get that, for any $0 \leq j \leq i-1$, $2=$ velocit $_{0} \geq$ velocity $_{j}>\alpha$. Thus, $\frac{2+4 * \text { velocity }_{i-1}+14 * \text { velocit }_{i-1}^{2}}{\left(\text { velocity }_{i-1}-1\right)^{2}}<\left\lceil\frac{20}{(\alpha-1)^{2}}\right\rceil$. Hence, we obtain that $1 / \beta_{i}<\left(\right.$ zoom $\left._{i}+\left\lceil\frac{20}{(\alpha-1)^{2}}\right\rceil\right) /$ zoom $_{i}<1+1 /\left(\frac{2}{\ln \left(\text { velocity }_{0} / \alpha\right)} * b^{i}\right)$.

For any $i, 0 \leq i \leq k$ :

$$
\begin{aligned}
2 & >1-(1 / b)^{k} \\
\ln \left(\text { velocit }_{0} / \alpha\right) & \left.>\frac{\ln \left(\text { velocit } y_{0} / \alpha\right)}{2} * \frac{1 / b-(1 / b)^{k+1}}{1-1 / b} \quad \text { (because } b>2\right) \\
& >\sum_{1 \leq j \leq i} 1 /\left(\frac{2}{\ln (\text { velocity } / \alpha)} * b^{j}\right) \\
& \geq \sum_{1 \leq j \leq i} \ln \left(1+1 /\left(\frac{2}{\ln (\text { velocity } / \alpha)} * b^{j}\right)\right) \quad(\text { because } x \geq \ln (1+x)) \\
& =\ln \left(\prod_{1 \leq j \leq i}\left(1+1 /\left(\frac{2}{\ln \left(\text { velocit } y_{0} / \alpha\right)} * b^{j}\right)\right)\right) \\
\text { velocit }_{0} / \alpha & >\prod_{1 \leq j \leq i}\left(1+1 /\left(\frac{2}{\ln \left(\text { velocit } y_{0} / \alpha\right)} * b^{j}\right)\right) \\
\text { velocit }_{0} / \alpha & >1 / \prod_{1 \leq j \leq i} \beta_{j}
\end{aligned}
$$

Lemma 2. Inequality 3 is satisfied. That is, for any $1 \leq i \leq k$, detour ${ }_{i}+4 *$ margin $_{i}+2<$ zoom $_{i}$ Proof. For any $1 \leq i \leq k$,

$$
\begin{aligned}
\text { detour }_{i}+4 * \text { margin }_{i}+2 & <2 *\left(\frac{\left(2 *\left(\frac{4+\text { velocit }_{i-1}}{\text { velocit }_{i-1}-1}+1\right)+2\right) \text { velocity }_{i-1}}{\text { velocity }_{i-1}-1}+1\right)+4\left(\frac{4+\text { velocit }_{i-1}}{\text { velocit }_{i-1}-1}+1\right)+2 \\
= & \frac{24 * \text { velocity }_{i-1}^{2}+4 * \text { velocit }_{i-1}-8}{\left(\text { velocity }_{i-1}-1\right)^{2}} \\
& <\frac{20}{(\alpha-1)^{2}} \quad(\text { because of Lemma } 1) \\
& <\text { zoom }_{i}=\frac{20}{(\alpha-1)^{2}} * \frac{2}{\ln \left(\text { velocit }_{0} / \alpha\right)} * b^{i} \\
& \left(\text { because } b>\max \left\{2, \frac{\ln \left(\text { velocit }_{0} / \alpha\right)}{2}\right\}\right)
\end{aligned}
$$

Both previous Lemmata allow us to prove the following:

Lemma 3. Let $i, 1 \leq i \leq k$. Let us assume that the robber occupies an $i$-nice position in a level-i subgrid $R^{i}$ in $G$. Moreover, let us assume that, all of the cops $\operatorname{cop}_{j}, j>i$, permanently remain outside around $\left(R^{i}\right)$. Let $D^{i}$ be any level-i subgrid adjacent to $R^{i}$.

The strategy described in section 2.2 ensures that

1. the robber reaches an $i$-nice position in $D^{i}$ in at most time ${ }_{i}<$ size $_{i}$ steps

2. cop $_{i}$ remains outside around $\left(R^{i-1}\right)$ during the whole game. 
Proof. The proof is by induction on $i$. W.l.o.g., let us assume that $D^{i}$ is below $R^{i}$. Recall that a square denotes a $(i-1)$-subgrid of $R^{i}$ or $D^{i}$. Also, $R_{s}^{i-1}$ denotes the square occupied by the robber at step $s$.

Let us consider the three possible strategies described in section 2.2.

- The robber goes down from its current $(i-1)$-nice position in square $R_{s}^{i-1}$ to an $(i-1)$ nice position to the below-neighbor of $R_{s}^{i-1}$, until $\operatorname{margin}\left(R_{s}^{i-1}\right)$ is a subgraph of $D^{i}$ (cf. Figure 1(a)).

Note that it is possible to apply the induction hypothesis because the robber occupies an $(i-1)$-nice position, all of the cops $\operatorname{cop}_{j}, j>i$, remain outside $\operatorname{around}\left(R^{i}\right) \supset \operatorname{around}\left(R_{s}^{i-1}\right)$ and we prove below that $\operatorname{cop}_{i}$ remains outside $\operatorname{around}\left(R_{s}^{i-1}\right)$.

First, by applying the induction hypothesis or straightforwardly if $i=1$, this strategy performs in at most $z o o m_{i} * t_{i m e} e_{i-1}$ steps. By equation 6, zoom $i * t_{i m e_{i-1}}<$ time $_{i}$.

This strategy is performed either if $\operatorname{cop}_{i}$ never blocks a side of $\operatorname{margin}\left(R_{s}^{i-1}\right)$, or $\operatorname{cop}_{i}$ blocks the above-side or an above-corner of $\operatorname{margin}\left(R_{s}^{i-1}\right)$. If $\operatorname{cop}_{i}$ never blocks a side of $\operatorname{margin}\left(R_{s}^{i-1}\right)$, the Lemma holds trivially. Let us assume the opposite.

If $i=1, t_{0}=1$ step is sufficient for the robber to go from one square to another (because size $_{0}=2$ and velocity $=2$ ), whereas cop $_{i}$ need at least size $e_{0}=2$ steps to performs the same displacement. Therefore, $\operatorname{cop}_{i}$ will never enter $\operatorname{margin}\left(R_{s}^{i-1}\right)$. If $i>1$, let us apply the induction hypothesis. $t_{i-1}$ step are sufficient for the robber to go from one square to another, whereas $\operatorname{cop}_{i}$ need at least $s_{i z e_{i-1}}$ steps to performs the same displacement. By Lemma 1, size $_{i-1}>t_{i-1}$. Therefore, each time the robber moves to a new square $R_{s}^{i-1}, \operatorname{cop}_{i}$ is outside of $\operatorname{margin}\left(R_{s}^{i-1}\right)$. In particular, this is the case at the step when $\operatorname{margin}\left(R_{s}^{i-1}\right)$ is contained in $D^{i}$ for the first time.

- The robber goes down from its current $(i-1)$-nice position in square $R_{s}^{i-1}$ to an $(i-1)$ nice position in the below-neighbor of $R_{s}^{i-1}$. After the first step when $\operatorname{margin}\left(R_{s}^{i-1}\right)$ is a subgraph of $D^{i}$, the robber continues going down along a path of $d_{i}$ extra squares in $D^{i}$ (cf. Figure 1(b)).

- First, by applying the induction hypothesis or straightforwardly if $i=1$, this strategy performs in at most $\left(z o o m_{i}+d_{i}\right) * t_{i m e} e_{i-1}$ steps. By equation $6,\left(z o o m_{i}+d_{i}\right) * t_{i m e} e_{i-1}<$ time $_{i}$.

This strategy is performed if there is a step $f$ of the game, when cop $_{i}$ blocks the left or the right-side or a below-corner of $\operatorname{margin}\left(R_{f}^{i-1}\right)$. Let $C_{f}^{i-1}$ be the square occupied by $\operatorname{cop}_{i}$ at this step. Note that $\left|a b s_{R_{f}^{i-1}}-a b s_{C_{f}^{i-1}}\right|=\operatorname{margin}_{i}+1$, and $\operatorname{ord}_{C_{f}^{i-1}} \geq \operatorname{ord}_{R_{f}^{i-1}}-\operatorname{margin}_{i}-1$.

- Let $s$ be some step of the game, and let us prove that $\operatorname{cop}_{i}$ cannot enter $\operatorname{around}\left(R_{s}^{i-1}\right)$ while the robber is occupying it. Indeed, by applying the induction hypothesis or straightforwardly if $i=1$, the robber will leave the square $R_{s}^{i-1}$ in at most $\operatorname{ord}_{R_{f}^{i-1}}-\operatorname{ord}_{R_{s}^{i-1}}+$ 1) time $_{i-1}$ steps after $f$. Two cases must be considered.

If $\operatorname{ord}_{R_{s}^{i-1}} \geq \operatorname{ord}_{R_{f}^{i-1}}-\operatorname{margin}_{i}-3$, the distance between $C_{f}^{i-1}$ and $R_{s}^{i-1}$, is at least $\left(\left|a b s_{R_{s}^{i-1}}-a b s_{C_{f}^{i-1}}\right|-2\right)$ (this distance is minimum for $\operatorname{ord}_{C_{f}^{i-1}}=\operatorname{ord}_{R_{s}^{i-1}}$ ). Therefore, $\operatorname{cop}_{i}$ requires at least $\left(\left|a b s_{R_{s}^{i-1}}-a b s_{C_{f}^{i-1}}\right|-2\right) * \operatorname{size}_{i-1}=\left(\operatorname{margin}_{i}-1\right) * \operatorname{size}_{i-1}$ steps to enter $\operatorname{around}\left(R_{s}^{i-1}\right)$. In this case, the robber leaves $R_{s}^{i-1}$ in at most $\left(\operatorname{margin}_{i}+4\right) *$ time $_{i-1}$ steps. By Inequality $1, \operatorname{cop}_{i}$ cannot enter around $\left(R_{s}^{i-1}\right)$ while the robber is occupying it (cf. Figure 2(a)).

Otherwise, the distance that $\operatorname{cop}_{i}$ must cover in order to enter $\operatorname{around}\left(R_{s}^{i-1}\right)$ is minimum when $\operatorname{ord}_{C_{f}^{i-1}}$ is minimum, that is, ord $C_{f}^{i-1}=\operatorname{ord}_{R_{f}^{i-1}}-\operatorname{margin}_{i}-1$. In this case, cop $_{i}$ requires at least $\left(\left|a b s_{C_{f}^{i-1}}-a b s_{R_{s}^{i-1}}\right|-2+\operatorname{ord}_{C_{f}^{i-1}}-\operatorname{ord}_{R_{s}^{i-1}}-2\right) * \operatorname{size}_{i-1}=\left(\operatorname{margin}_{i}-\right.$ 
$\left.1+\operatorname{ord}_{R_{f}^{i-1}}-\operatorname{margin}_{i}-1-\operatorname{ord}_{R_{s}^{i-1}}-2\right) * \operatorname{size}_{i-1}=\left(\operatorname{ord}_{R_{f}^{i-1}}-\operatorname{ord}_{R_{s}^{i-1}}-4\right) * \operatorname{size}_{i-1}$ steps to enter around $\left(R_{s}^{i-1}\right)$. By Inequality 1 and Lemma $1\left(\right.$ size $\left._{i-1}>t_{i-1}\right)$, we get $\left(\operatorname{ord}_{R_{f}^{i-1}}-\operatorname{ord}_{\left.R_{s}^{i-1}-4\right) * \text { size }_{i-1}}=\left(\operatorname{ord}_{R_{f}^{i-1}}-\operatorname{ord}_{R_{s}^{i-1}}-\operatorname{margin}-3\right) *\right.$ size $_{i-1}+\left(\operatorname{margin}_{-}\right.$ $1) *$ size $_{i-1}>\left(\operatorname{ord}_{R_{f}^{i-1}}-\operatorname{ord}_{R_{s}^{i-1}}-\right.$ margin $\left._{-3}\right) *$ time $_{i-1}+\left(\operatorname{margin}_{i}+4\right) * \operatorname{time}_{i-1}=$ $\left.\operatorname{ord}_{R_{f}^{i-1}}-\operatorname{ord}_{R_{s}^{i-1}}+1\right) * t i m e_{i-1}$. Again, $\operatorname{cop}_{i}$ cannot enter around $\left(R_{s}^{i-1}\right)$ while the robber is occupying it.

This concludes the proof of the fact that $\operatorname{cop}_{i}$ remains outside around $\left(R^{i-1}\right)$ during the whole game.

- It remains to prove that the robber reaches an $i$-nice position in $D^{i}$. That is, we prove that $\operatorname{cop}_{i}$ is above $\operatorname{margin}\left(R_{h}^{i-1}\right)$ at the last step $h$ of the game, and $\operatorname{margin}\left(R_{h}^{i-1}\right)$ is a subgraph of $D^{i}$. Let top (resp., right) be the greatest ordinate (resp., abscissa) of the squares in $D^{i}$. Note that, ord $R_{h}^{i-1}=$ top $-\operatorname{margin}_{i}-d_{i}$ and $a b s_{R_{h}^{i-1}}=a b s_{R_{f}^{i-1}}$.

By Lemma 2, $d_{i}<$ zoom $_{i} / 2$. Thus, top $-\operatorname{margin}_{i}>\operatorname{ord}_{R_{h}^{i-1}}>$ top - zoom $_{i}+\operatorname{margin}_{i}$. Moreover, margin $\left(R_{f}^{i-1}\right)$ is a subgraph of $R^{i}$, thus right-zoom ${ }_{i}+\operatorname{margin}_{i}<a b s_{R_{h}^{i-1}}<$ right - $\operatorname{margin}_{i}$. Therefore, $\operatorname{margin}\left(R_{h}^{i-1}\right)$ is a subgraph of $D^{i}$.

The distance that $\operatorname{cop}_{i}$ must cover in order to enter $\operatorname{margin}\left(R_{s}^{i-1}\right)$ is minimum when $\operatorname{ord}_{C_{f}^{i-1}}$ is minimum, that is, $\operatorname{ord}_{C_{f}^{i-1}}=\operatorname{ord}_{R_{f}^{i-1}}-\operatorname{margin}_{i}-1$. In this case, $\operatorname{cop}_{i}$ requires at least $\left(\left|a b s_{C_{f}^{i-1}}-a b s_{R_{h}^{i-1}}\right|-2+\operatorname{ord}_{C_{f}^{i-1}}-\operatorname{ord}_{R_{h}^{i-1}}-2\right) * \operatorname{size}_{i-1}=\left(\operatorname{margin}_{i}-1+\right.$ $\left.\operatorname{ord}_{R_{f}^{i-1}}-\operatorname{margin}_{i}-1-\operatorname{ord}_{R_{h}^{i-1}}-2\right) * \operatorname{size}_{i-1}=\left(\operatorname{ord}_{R_{f}^{i-1}}-\operatorname{ord}_{R_{h}^{i-1}}-4\right) * \operatorname{size}_{i-1}$ steps to enter around $\left(R_{s}^{i-1}\right)$. By Inequality 2 and Lemma $1\left(\right.$ size $\left._{i-1}>t_{i-1}\right)$, we get $\left(\operatorname{ord}_{R_{f}^{i-1}}-\operatorname{ord}_{R_{s}^{i-1}-4}\right) * \operatorname{size}_{i-1}=\left(\operatorname{ord}_{R_{f}^{i-1}}-\operatorname{ord}_{R_{s}^{i-1}}-d_{i}+2 * \operatorname{margin}_{i}+2\right) * \operatorname{size}_{i-1}+\left(d_{i}-\right.$ $\left.2 * \operatorname{margin}_{i}-2\right) *$ size $_{i-1}>\left(\operatorname{ord}_{R_{f}^{i-1}}-\operatorname{ord}_{R_{s}^{i-1}}-d_{i}+2 * \operatorname{margin}_{i}+2\right) *$ time $_{i-1}+d_{i} *$ time $_{i-1}=$ $\left(\operatorname{ord}_{R_{f}^{i-1}}-\operatorname{ord}_{R_{s}^{i-1}}+2 * \operatorname{margin}_{i}+2\right) *$ time $_{i-1}$. Moreover, by applying the induction hypothesis or straightforwardly if $i=1$, at most $\left(\operatorname{ord}_{R_{f}^{i-1}}-\operatorname{ord}_{R_{h}^{i-1}}\right)$ time $_{i-1}$ steps are sufficient for the robber to reach an $(i-1)$-nice position in the square $R_{h}^{i-1}$. Therefore, $\operatorname{cop}_{i}$ cannot enter $\operatorname{margin}\left(R_{h}^{i-1}\right)$ while the robber is occupying it.

This concludes the proof of the fact that the robber reaches an $i$-nice position in $D^{i}$.

- The robber goes down from its current $(i-1)$-nice position in square $R_{s}^{i-1}$ to an $(i-1)$-nice position to the below-neighbor of $R_{s}^{i-1}$. At some step $f, c_{i}$ is blocking the side below $\operatorname{margin}\left(R_{f}^{i-1}\right)$. The robber chooses the right side, if $R_{f}^{i-1}$ is closest to this side of $R^{i}$, and the left side otherwise. W.l.o.g, let the robber choose the right side. Then, the robber first goes to the right, along a path of $d_{i}$ squares. Let $R_{r}^{i-1}$ be the last of these squares, at which the robber arrives at step $r$. Starting from step $r$, the robber goes down, and after the first step when margin $\left(R_{s}^{i-1}\right)$ is a subgraph of $D^{i}$, the robber continues going down along a path of $d_{i}$ extra squares in $D^{i}$ (cf. Figure 1(c)).

First, by applying the induction hypothesis or straightforwardly if $i=1$, this strategy performs in at at most $\left(\right.$ zoom $_{i}+$ detour $\left._{i}\right) *$ time $_{i-1}$ steps. By equation $6,\left(\right.$ zoom $_{i}+$ detour $\left._{i}\right) *$ time $_{i-1}=$ time $_{i}$.

Let right be the greatest abscissa of the squares in $D^{i}$, and let $h$ be the last step of the game. Note that, by Lemma $2, a b s_{R_{r}^{i-1}}<$ right-margin. Therefore, by the same analysis as in the last item of the previous case, $\operatorname{margin}\left(R_{h}^{i-1}\right)$ is a subgraph of $D^{i}$.

Then, we prove that $\operatorname{cop}_{i}$ remains outside around $\left(R_{s}^{i-1}\right)$ for any square $R_{s}^{i-1}$ on the path between $R_{f}^{i-1}$ and $R_{r}^{i-1}$. For this purpose, it is sufficient to observe that the configuration is similar to the previous strategy, by reflecting the grid along the $R_{s}^{i-1}-C_{s}^{i-1}$ axis. Moreover, 
this observation proves that when the robber arrives in $R_{r}^{i-1}, c o p_{i}$ neither can stand inside $\operatorname{margin}\left(R_{r}^{i-1}\right)$, nor block the bottom side of $\operatorname{margin}\left(R_{r}^{i-1}\right)$.

Let us show that $\operatorname{cop}_{i}$ cannot block the bottom side of $\operatorname{margin}\left(R_{s}^{i-1}\right)$ for any remaining step $s$ of the game. Indeed, the distance that $\operatorname{cop}_{i}$ must cover in order to block the bottom side of $\operatorname{margin}\left(R_{s}^{i-1}\right)$ is minimum when $\operatorname{ord}_{C_{f}^{i-1}}=\operatorname{ord}_{R_{f}^{i-1}}-\operatorname{margin}_{i}-1$ and $a b s_{C_{f}^{i-1}}=$ $a b s_{R_{f}^{i-1}}+\operatorname{margin}_{i}$. In this case, $\operatorname{cop}_{i}$ requires at least $S=\left(a b s_{R_{s}^{i-1}}-a b s_{C_{f}^{i-1}}-\operatorname{margin}_{i}-\right.$ $\left.1+\operatorname{ord}_{C_{f}^{i-1}}-\operatorname{ord}_{R_{s}^{i-1}}+\operatorname{margin}_{i}\right) * \operatorname{size}_{i-1}$ steps to block the bottom side of $\operatorname{margin}\left(R_{s}^{i-1}\right)$. By Inequality 2 and Lemma $1, S=\left(a b s_{R_{s}^{i-1}}-a b s_{R_{f}^{i-1}}-2 * \operatorname{margin}_{i}-2+\operatorname{ord}_{R_{f}^{i-1}}-\right.$ $\left.\operatorname{ord}_{R_{s}^{i-1}}\right) * \operatorname{size}_{i-1}=\left(d_{i}-2 * \operatorname{margin}_{i}-2\right) * \operatorname{size}_{i-1}+\left(\operatorname{ord}_{R_{f}^{i-1}}-\operatorname{ord}_{R_{s}^{i-1}}\right) * \operatorname{size}_{i-1}>$ $d_{i} *$ time $\left._{i-1}+\operatorname{ord}_{R_{f}^{i-1}}-\operatorname{ord}_{R_{s}^{i-1}}\right) * \operatorname{time}_{i-1}>\left(d_{i}+\operatorname{ord}_{R_{f}^{i-1}}-\operatorname{ord}_{R_{s}^{i-1}}\right) *$ time $_{i-1}$ which is the upper bound on the number of steps after $f$ required to leave $R_{s}^{i-1}$ (by applying the induction hypothesis). Hence, $\operatorname{cop}_{i}$ cannot block the bottom side of $\operatorname{margin}\left(R_{s}^{i-1}\right)$ for any square $R_{s}^{i-1}$ on the path between $R_{r}^{i-1}$ and $R_{h}^{i-1}$.

Therefore, the configuration is the same as in the previous cases and the lemma holds.

This concludes the proof of the lemma and of Theorem 1

We are now able to prove Theorem 1

Proof. More precisely, we prove that, for any $k \geq 1$, one robber with speed velocity $y_{0}=2$ can infinitely evade $k$ cops with speed one in any grid of size more than $4 a^{k} b^{k(k+1) / 2}$, where $a$ and $b$ are defined as previously.

Let $G$ be the grid of size $2 *$ size $_{k}=2 *$ size $_{0} * \prod_{1 \leq i \leq k} z o o m_{i}=4 * a^{k} * b^{k(k+1) / 2}$. Note that, if one robber can infinitely evade $k$ cops in $G$, it can perform the same strategy and evade $k$ cops as well in any bigger grid. It remains to prove that the strategy described in Section 2.2 enables the robber to infinitely evade $k$ cops in $G$.

Now, let us assume that $k$ cops are placed on vertices of $G$. $G$ is divided into 4 vertex-disjoint subgrids of size $s_{i z e_{k}}$ (i.e., level- $k$ subgrids). Let us fix an ordering of the cops $\left(\operatorname{cop}_{1}, \ldots, \operatorname{cop}_{k}\right)$. Choose one of the level- $k$ subgrids not occupied by $c o p_{k}$, and denote it by $R^{k}$. Notice that, by Equation $3, R^{k}$ contains at least four $(k-1)$-subgrids $R_{1}^{k-1}, \ldots, R_{1}^{k-1}$ such that $\operatorname{margin}\left(R_{i}^{k-1}\right)$, $1 \leq i \leq 4$, are disjoint and entirely contained in $R^{k}$. Any position inside these subgrids is nice at level $k$. Recursively, choose one not occupied by $\operatorname{cop}_{k-1}$ to be $R^{k-1}$, and proceed until finding $R^{0}$. Any position inside $R^{0}$ is $k$-nice and we may pick it as the initial position for the robber. The top level strategy consists in traversing the four level- $k$ subgrids of $G$ along the cycle given by their adjacencies. Lemma 3 (by taking $i=k$ ) proves that, starting from a $k$-nice position in some level- $k$ subgrid $R^{k}$, the robber can reach a $k$-nice position in any level- $k$ subgrid adjacent to $R^{k}$, without being caught by the cops. By repeating this process infinitely, the robber can infinitely evade $k$ cops in $G$, which proves Theorem 1 .

Corollary 1. For any grid $G$ of size $n$, and for any $1 \leq p<q, c_{p, q}(G)>\Omega(\sqrt{\log (n)})$.

Proof. Let $0<\alpha<1$. By setting, size $0=q, a=\left\lceil\frac{2+4 q+14 q^{2}}{(p+\alpha-1)^{2}}\right\rceil$, and $b>\max \left\{2, \frac{\ln (q /(p+\alpha))}{2}\right\}$, the proof the corollary follows the proof of Theorem 1.

\section{Fast robber cop-number with respect to graph containment}

We have seen that the number of cops needed to capture a fast robber in a grid $G$ may be arbitrarily large. It would be interesting to see if a high value of the cop-number of a planar 
graph $H$ is related to a large grid $G$ somehow contained in $H$. On the negative side, the classical transformations of edge removal, vertex removal, and edge contraction do not preserve bounded cop-number. Moreover, there are graphs of arbitrarily large tree-width [Bod98] (that is, somehow containing a large grid) and cop-number two.

Proposition 2. For any $k \geq 1$, there is a planar graph $H$ with $c_{1,2}(H) \leq 2$, such that a graph $G$ with $c_{1,2}(G) \geq k$ can be obtained from $H$ by contracting edges (resp., by removing edges, resp., by removing vertices).

Proof. Let $k \geq 1$. Let $G$ be the grid of size $n \geq f(k)$ (cf. definitions in Section 2). For the following descriptions, let us fix a planar embedding with vertices being the positive integer points of $n \times n$ square. Let $S D_{\ell}(G)$ be the graph obtained by subdividing each edge of $G$ to create a path of length $\ell$. Note that, the reasoning used to prove Theorem 1 can be easily extended to show that the cop-number of $S D_{\ell}(G)$ also is at least $k$.

Let us call the column (vertical path) of vertices of abscissa equal $d=\lceil n / 2\rceil$ the backbone. Let $H$ be the graph obtained from $S D_{6 d}(G)$ by recursively contracting edges incident to a vertex of degree two on a line (horizontal path) or on the backbone. In other words, $H$ results from the graph $G$ by replacing all "vertical" edges, except for these on the backbone, with paths of length $6 d$. Let us propose a strategy that allows to capture the robber in $H$ with just two cops. Put these cops, denoted $c, c^{\prime}$, on the intersections of the backbone with the lines of ordinate $1 \leq o<n$ and $o^{\prime}=o+1$. The robber chooses its position, and then we start moving towards it.

Suppose that the robber occupies a position of ordinate $o_{r}$ between $o^{\prime}$ and $o^{\prime}-1 / 2$. Start moving $c^{\prime}$ towards the robber, along the line $o^{\prime}$. At some point, the robber needs to take a long path towards the line above or the line below - otherwise $c^{\prime}$ captures it at the border of the grid. Suppose that the robber takes the column of abscissa $a$, between lines $o^{\prime}$ and $o$. Then $c^{\prime}$ continues moving to the intersection of line $o^{\prime}$ with $P$. When $c^{\prime}$ reaches this intersection, the robber has covered a distance of at most $4 d$ downwards $P$. Indeed, it takes at most $d$ steps for $c^{\prime}$ to reach the intersection, and the initial position of the robber had ordinate at least $o^{\prime}-1 / 2$. Now $c$ moves to the other end of $P$, along the line $o$ - this distance is at most $d$, so he arrives before the robber can reach this point. The robber is blocked on an induced path with both ends guarded by cops, thus the cops can approach and eventually capture it. So, suppose the robber takes the column of abscissa $a$ on the way towards the line $o^{\prime}+1$. Like in the previous case, $c^{\prime}$ blocks the intersection of the line $o^{\prime}$ with the column $a$ in at most $d$ steps. Then, $c$ first crosses two edges up along the backbone and then approaches the intersection of $P$ with the line $o^{\prime}+1$, along this line. It takes at most $d+2$ steps. By a similar reasoning to that of the previous case, they manage to block the ends of $P$ and eventually capture the robber. If the robber is not at a vertex of ordinate between $o$ and $o^{\prime}$, let the cops approach it along the backbone, occupying two consecutive vertices at each step. This way we recreate the configuration described above, at the top (or bottom) line of the grid, at the latest. Thus, two cops are sufficient to capture the robber in $H$.

Therefore, with contraction of some edges of $S D_{6 d}(G)$, the cop-number drops from at least $k$ down to 2 . On the other hand, by contracting all edges incident to a vertex of degree two in $H$ we obtain $G$, so the cop-number drastically goes up again.

Now let the column of vertices of abscissa equal $d$ be the backbone of $G$, and let us consider $S D_{12 d}(G)$. For every edge of a line or of the backbone of $G$ add a path of length two between the corresponding vertices in $S D_{12 d}(G)$, and denote the resulting graph by $H$. Notice that a strategy analogous to the one described above also allows to capture the robber with just two cops in $H$, i.e., $c_{1,2}(H) \leq 2$. By removing (in $H$ ) these auxiliary vertices or one edge incident to each of them, we obtain a graph $G$ in which it is as difficult to capture the robber as in 
$S D_{12 d}(G)$, i.e., $c_{1,2}(G) \geq k$. On the other hand, by removing some vertices or removing some edges from $S D_{12 d}(G)$ we can create a tree, thus a graph of cop-number two.

Let $G$ be a distance-hereditary subgraph of a planar graph $H$, i.e., the distance between any two vertices in $G$ is the same as the corresponding distance in $H$. Even in this setting, the cop-number of $G$ may be higher than that of $H$. Indeed, take $G$ to be the graph $C_{4}$, a cycle on four vertices. Add a universal vertex $u$ to $G$ and denote the resulting graph by $H$. Catching the robber in $C_{4}$ takes 2 cops, whereas just one cop placed on $u$ in $H$ does the job in one move. On the other hand, take $G^{\prime}$ to be $K_{2}$. This time, taking a distance preserving induced subgraph $G^{\prime}$ of $G$ lowers the cop-number.

Nevertheless, we can define a larger family of planar graphs of high cop-number than the grids themselves.

Theorem 2. Let $H$ be a planar graph containing a grid $G$ of size $4 *$ size $_{k}$ as an induced subgraph, then $c_{1,2}(H) \geq k$.

Proof. Notice that if, for each of the vertices of degree 2 in $G$ (the "corners"), we contract one incident edge, then we obtain a 3-connected planar graph. By a theorem of Whitney (see Theorem 4.3.2 of [Die05]), a 3-connected planar graph has only one embedding into the sphere, modulo topological equivalence. So, the embedding of $G$ is also unique, and any embedding of the whole $H$ has to respect it. That means that, for any two vertices $u, v$ of $G$, and for any path $P$ between $u$ and $v$ such that any internal vertex of $P$ belongs to $H \backslash G$, whatever be the embedding of $H$ into the sphere, only two cases may occur, otherwise there would have been a crossing between an edge of $P$ and an edge of $G$. Either $P$ belongs to the external face of the embedding of $G$, or $P$ belongs to the face limited by an induced 4-vertices cycle $C$ of $G$, such that $u, v \in V(C)$. In the latter case, since $G$ is an induced subgraph, $P$ contains at least one vertex in $H \backslash G$.

Notice that $G$ can be partitioned into sixteen subgrids of size size $e_{k}$. Consider the four of them that are in the center of this partition, and together form a subgrid of size $2 *$ size $_{k}$. Denote it by $G^{\prime}$. Because $H$ has to respect the embedding of $G$, it cannot be a path $P$ in $H \backslash G^{\prime}$ between two vertices of $G^{\prime}$ strictly shorter than the paths in $G^{\prime}$.

The escape strategy used in the proof of Theorem 1 can be easily adopted to $H$, with the robber restricted to stay in $G^{\prime}$. The reasoning used in the proof can be easily extended to see that the robber can find a $k$-nice initial position, and keep moving in order to keep his position $k$-nice forever. The arguments used to show that the cops cannot get too close to the robber applying our strategy remain valid, since $G^{\prime}$ preserves the distances. In other words, there are no "short-cuts" available in $H$. Notice that it is a particular property of our escape strategy, that the absence of short-cuts in $H \backslash G^{\prime}$ ensures that the robber can still escape in $H$. Indeed, the strategy of the robber is mainly based on the distance between the robber and the cops. 


\section{References}

[AF84] Martin Aigner and Michael Fromme. A game of cops and robbers. Discrete Applied Mathematics, 8:1-12, 1984.

[AF88] Richard P. Anstee and Martin Farber. On bridged graphs and cop-win graphs. J. Comb. Theory, Ser. $B, 44(1): 22-28,1988$.

[Als04] Brian Alspach. Searching and sweeping graphs: a brief survey. In Le Matematiche, pages 5-37, 2004.

[And84] Thomas Andreae. Note on a pursuit game played on graphs. Discrete Applied Mathematics, 9:111-115, 1984.

[And86] Thomas Andreae. On a pursuit game played on graphs for which a minor is excluded. J. Comb. Theory, Ser. B, 41(1):37-47, 1986.

[BI93] Alessandro Berarducci and Benedetto Intrigila. On the cop number of a graph. Adv. in Applied Math., 14:389-403, 1993.

[Bod98] Hans L. Bodlaender. A partial -arboretum of graphs with bounded treewidth. Theor. Comput. Sci., 209(1-2):1-45, 1998.

[Che97] Victor Chepoi. Bridged graphs are cop-win graphs: An algorithmic proof. J. Comb. Theory, Ser. B, 69(1):97-100, 1997.

[Die05] Reinhard Diestel. Graph Theory, third edition. Springer-Verlag, 2005.

[Far87] Martin Farber. Bridged graphs and geodesic convexity. Discrete Applied Mathematics, 66:249-257, 1987.

[Fra87] Peter Frankl. Cops and robbers in graphs with large girth and cayley graphs. Discrete Applied Mathematics, 17:301-305, 1987.

[GR95] Arthur S. Goldstein and Edward M. Reingold. The complexity of pursuit on a graph. Theor. Comput. Sci., 143(1):93-112, 1995.

[HM06] Gena Hahn and Gary MacGillivray. A note on $k$-cop, l-robber games on graphs. Discrete Math., 306:2492-2497, 2006.

[NW83] Richard J. Nowakowski and Peter Winkler. Vertex-to-vertex pursuit in a graph. Discrete Mathematics, 43:235-239, 1983.

[Qui83] Alain Quilliot. Thèse de doctorat d'état. PhD thesis, Université de Paris VI, France, 1983.

[Qui85] Alain Quilliot. A short note about pursuit games played on a graph with a given genus. J. Comb. Theory, Ser. B, 38(1):89-92, 1985.

[Sch01] Bernd S. W. Schröder. The copnumber of a graph is bounded by $\left\lfloor\frac{3}{2}\right.$ genus $\left.(g)\right\rfloor+3$. Trends Math., pages 243-263, 2001. 